\title{
Suprarenal solitary fibrous tumor associated with a NF1 gene mutation mimicking a kidney neoplasm: implications for surgical management
}

\author{
Giovanni Conzo ${ }^{1 *}$, Ernesto Tartaglia', Claudio Gambardella ${ }^{1}$, Claudio Mauriello ${ }^{1}$, Daniela Esposito ${ }^{1}$, \\ Massimo Mascolo², Daniela Russo², Gianfranca Stornaiuolo³, Giovan Battista Gaeta ${ }^{3}$ and Luigi Santini ${ }^{1}$
}

\begin{abstract}
Solitary fibrous tumor (SFT) is a rare spindle cell neoplasm, usually occurring in the pleura. Pararenal SFT, mimicking an adrenal gland or renal tumor, as here described, is extremely rare. We report a case of a right suprarenal SFT, incidentally discovered by abdominal ultrasound in a 54-year-old woman carrying a point neurofibromatosis 1 (NF1) gene mutation. Preoperative diagnostic work-up was ineffective in evaluating its origin, and an open radical right nephrectomy was therefore undertaken. Immunohistochemical assay showed a positivity for CD34, CD99 and BCl-2, so suggesting a diagnosis of SFT. According to our knowledge, the association between this type of tumor and NF1 gene mutation has never been described. In cases of pararenal tumors, a more detailed preoperative diagnosis could be useful to better plan the extension of resection, allowing, in selected cases, nephron-sparing surgery. More studies are needed to better analyze the relationship between NF1 gene mutation and SFT.
\end{abstract}

Keywords: Solitary fibrous tumor, Spindle cells, Renal neoplasm, Immunohistochemical assay, Neurofibromatosis gene mutation

\section{Background}

Solitary fibrous tumor (SFT) is a rare spindle cell neoplasm, firstly described in the1930s [1]. Prognosis seems to be favorable, but large series with long-term follow-up are still lacking. This kind of tumor, usually occurring in the pleura, the so called 'localized fibrous mesothelioma', has recently been described in different and multiple extrapleural sites including orbit, nasal cavity, breast, adrenal or thyroid gland, liver and lung [2]. Although the retroperitoneum is frequently involved, according to a computed search of the medical literature, only 39 cases of renal or pararenal SFT have been reported. Tumor size ranged from 2 to $25 \mathrm{~cm}$, and nephrectomy was the standard treatment. The origin of these neoplasms is still controversial $[3,4]$. They are considered as slow-growing tumors with a favorable prognosis, although malignant cases, showing locoregional recurrence or distant metastases, have been

\footnotetext{
* Correspondence: giovanni.conzo@unina2.it

'Department of Anesthesiologic, Surgical and Emergency Science VII Division of General Surgery, Second University of Naples Italy, Via Pansini 5, 80131 Naples, Italy

Full list of author information is available at the end of the article
}

reported. Neurofibromatosis type 1 (von Recklinghausen's disease-NF1) is caused by an alteration of the NF1 gene, a tumor suppressor located on the long arm of chromosome 17 (17q11.2) [5]. Loss of the gene function, due to a point mutation, leads to an increase in cell proliferation and to development of tumors [5].

We describe a right suprarenal SFT in a female patient carrying a NF1 gene mutation. Preoperative diagnostic work-up was unable to determine its primal tissue, and therefore an open radical right nephrectomy was performed. Only the immunohistochemical assay allowed a correct pathological diagnosis. To better evaluate the role of radical nephrectomy, in a case of pararenal tumor with no clear signs of renal infiltration, more detailed therapeutic guidelines could be useful to establish the recommended extension of resection. According to our literature search, this is the first case in which SFT was associated to a NF1 gene mutation.

\section{Case presentation}

In July 2012, a 52-year-old woman was referred to our observation for the presence of a solid mass in her right 


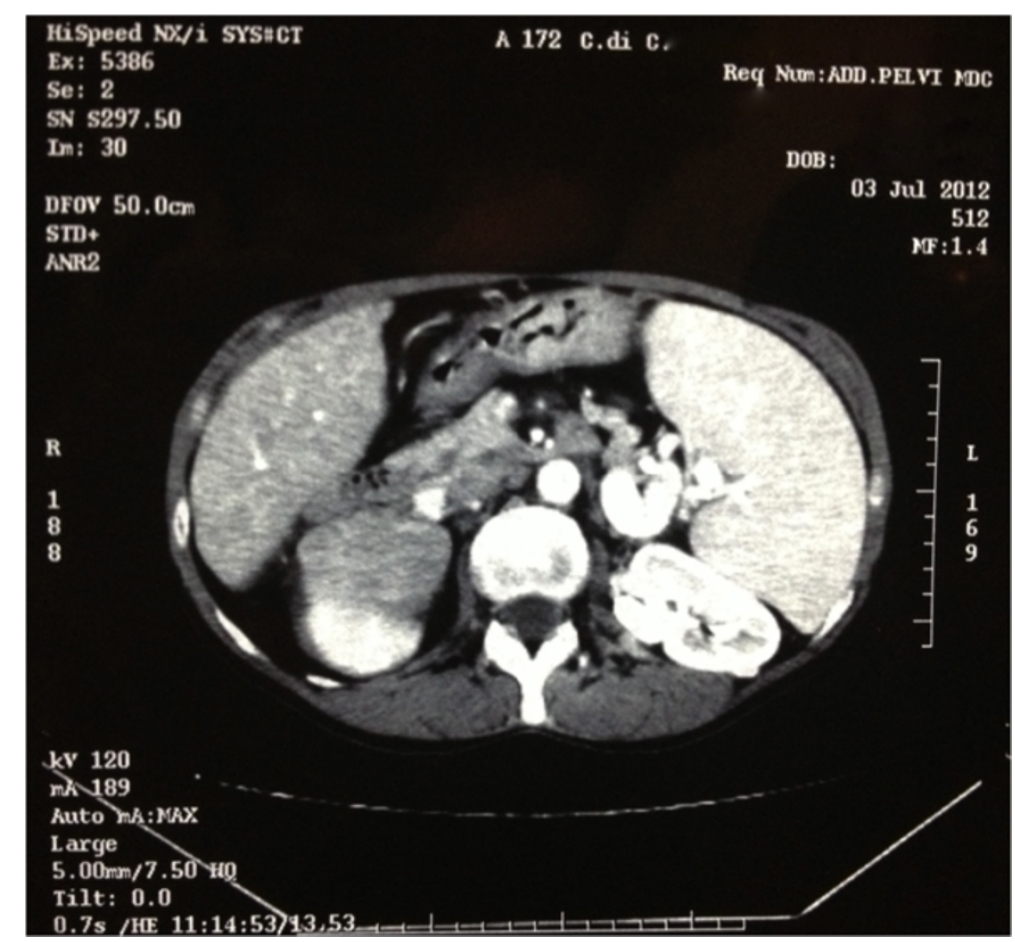

Figure 1 Computerized tomographic scan depicting large superior pole mass of the right kidney.

kidney, detected during a routine follow-up for chronic hepatitis B virus infection. Computed tomography scan showed a well-delineated, encapsulated tumor arising from the upper pole of the right kidney and measuring $96 \times 63 \mathrm{~mm}$, contrast-enhanced and with no evidence of vessel infiltration (Figure 1). The ipsilateral adrenal gland was not clearly distinguishable from the neoplasm, and its adrenal origin was apparent. Magnetic resonance imaging (MRI) scan showed a mass of low intensity on T1-weighted images and of irregular high intensity on T2-weighed images. Neither renal vein nor inferior vena cava thrombosis were present, and suspected enlarged lymph nodes were also not identified. Due to the presence of a daughter with a previous diagnosis of NF1 gene mutation, and the presence of small neurofibromas over the patient's body, a genetic test for NF1 using reverse transcription (RT)-PCR and high throughputdenaturing high performance liquid chromatography (HTDHPLC) was performed, confirming the diagnosis of von Recklinghausen's disease. Considering that a pheochromocytoma (PCC) has been identified in 0.1 to $5.7 \%$ of patients with von Recklinghausen's disease, a PCC was considered in the differential diagnosis [6]. The absence of clinical signs and of arterial pressure lability, associated with a normal level of urinary metanephrine concentrations and the absence of pathological uptake following MIBG (meta iodo benzyl guanidine) scintigraphy, allowed the exclusion of a PCC neoplasm. Since a diagnosis of suspected renal or adrenal gland mass was suspected, the patient underwent an explorative laparotomy. During the procedure, a large neoplasm, strongly adherent to the upper renal pole and to the ipsilateral suprarenal space, was observed and, presuming a renal cell carcinoma, we undertook a surgical resection including a radical right nephrectomy. No macroscopic enlarged lymph nodes were observed. The postoperative course was uneventful

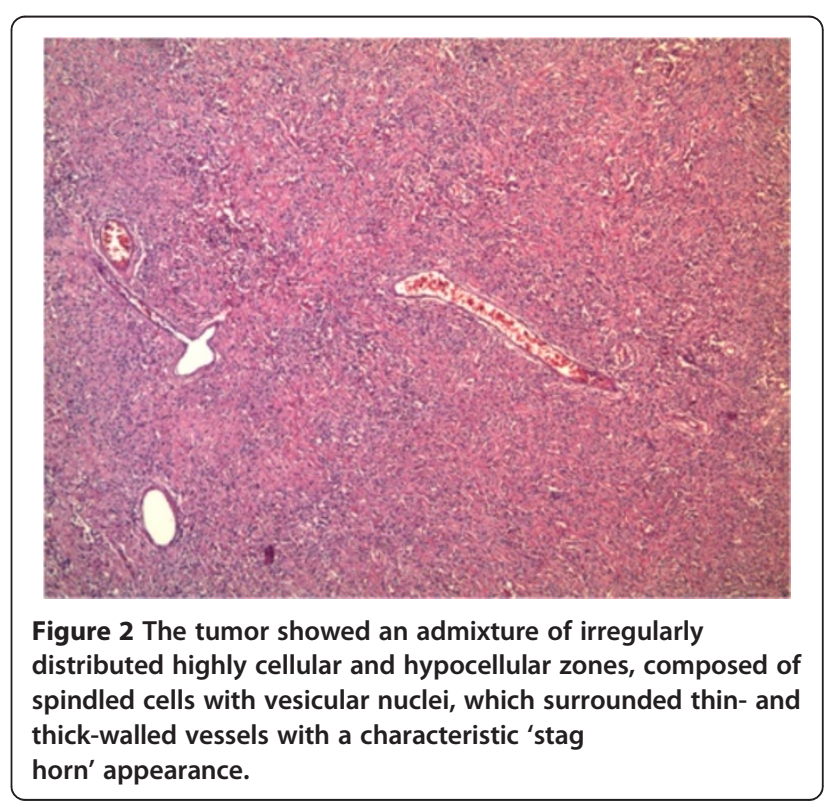


and the patient was discharged on postoperative day four. Diagnostic work-up was negative during the eight-month follow-up. The gross specimen included: right kidney, ureter, adrenal gland, perinephric tissue and a mass measuring $90 \times 60 \times 40 \mathrm{~mm}$ in overall dimensions. At microscopy, the tumor showed an admixture of irregularly

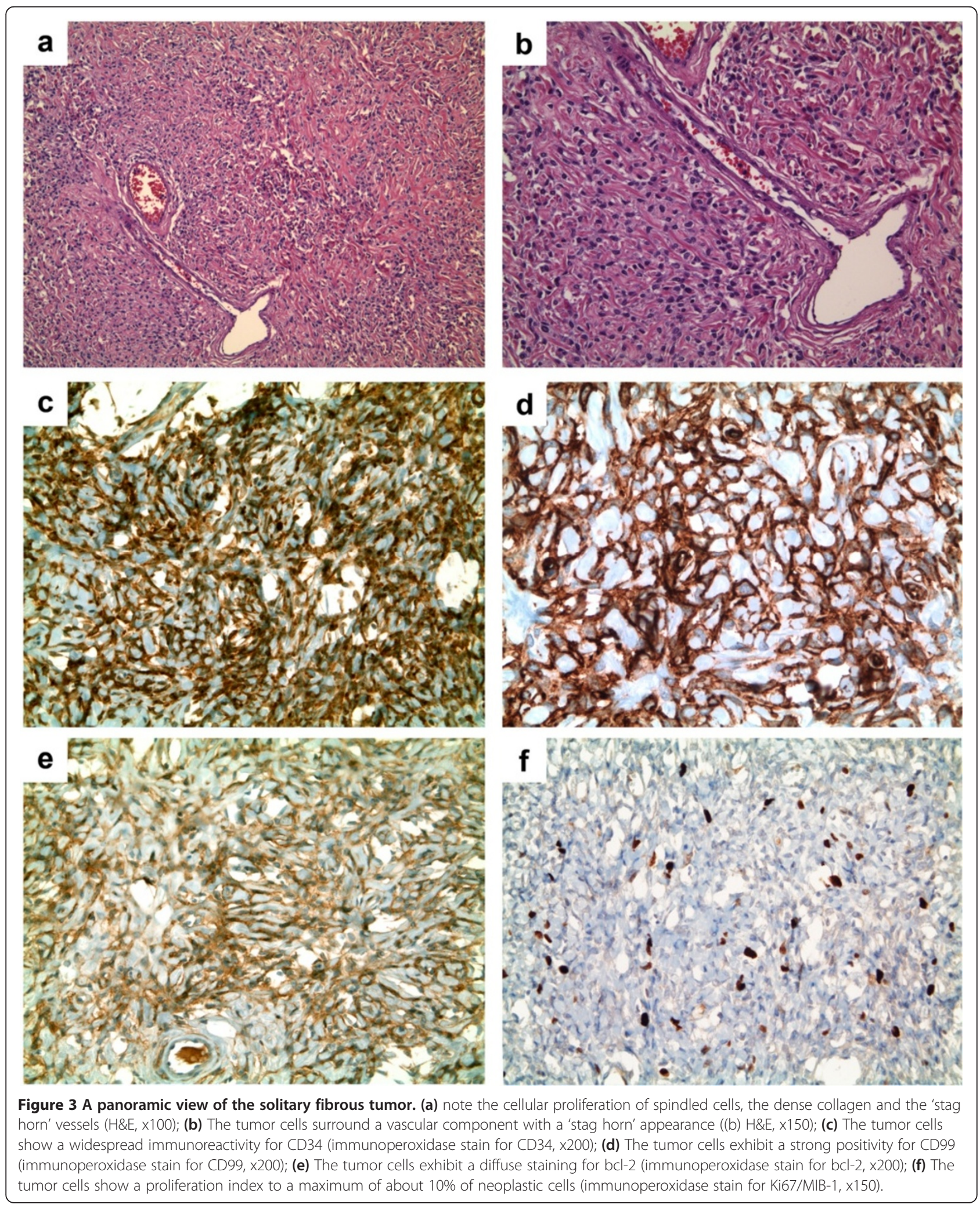


distributed highly cellular and hypocellular areas, composed of spindled cells with vesicular nuclei, which surrounded thin- and thick-walled vessels with a characteristic 'stag horn' appearance. A prominent hyalinized collagen was present (Figure 2). Most tumor cells expressed CD34, CD99, and bcl-2, whereas cytokeratin, CD117 (c-Kit), S100 protein and EMA were negative. The Ki-67 (MIB-1) staining, a marker of cellular proliferation, was irregularly expressed, exhibiting positivity in up to about $10 \%$ of neoplastic cells. Based on morphological features and immunohistochemical profile, a diagnosis of a solitary fibrous tumor was made (Figure 3).

\section{Conclusions}

In most cases of SFT, described as renal or pararenal tumor, it is very difficult to determine the true origin from the renal capsule [4], the interstitial tissue, or the peripelvic connective tissue. Therefore, in planning a therapeutic protocol, extension of resection may be controversial and 'classical' nephrectomy remains the most common treatment. In the reported case, only definitive pathology identified a well-encapsulated SFT not infiltrating the kidney and the ipsilateral adrenal gland, and radical nephrectomy could probably be considered an overtreatment. Histopathological examination, immunohistochemical and ultrastructural studies are the cornerstone of SFT diagnosis. Morphologically, it is characterized by spindle cell proliferation, and about $70 \%$ of cases expresses CD 34, CD99, and Bcl-2; only between 20 and $35 \%$ of cases are variably positive for epithelial membrane antigen and smooth muscle actin. Focal and limited reactivity of S-100 protein, cytokeratins and/or desmin has also occasionally been reported [6]. Strong CD34 reactivity is currently regarded as characteristic, and an indispensable finding in the diagnosis of SFT [7].

Since these tumors typically show hemangiopericytomatous patterns, differential diagnosis includes sarcomatoid renal cell carcinoma or renal adenoma and other benign spindle cell tumors such as angiomyolipoma, fibroma, or fibrosarcoma [4]. In presence of a NF1 gene mutation, neurofibroma should also be considered. SFT is a rare tumor sometimes mimicking renal cell or adrenal gland neoplasms [8,9], and it must be considered in cases of renal tumors comprising mesenchymal elements. In roughly 10 to $15 \%$ of cases, they behave aggressively, showing local recurrence or distant metastases [4]. According to England et al. [10], increased cellularity with crowded/overlapping nuclei, cellular pleomorphism, and a mitotic count of more than four figures per ten high-power fields are considered criteria for malignancy. However, clinical progression of tumors with a benign appearance cannot be predicted on histopathological basis, which can exhibit aggressive behavior, and vice versa. Therefore, all SFT patients need to be on long-term follow-up $[4,7]$. Turning to treatment, complete surgical resection is recommended [3], but because SFTs exhibit relative chemo-radio tumor resistance, no data are reported regarding the role of adjuvant treatment. In case of a preoperative suspicion of a well-encapsulated pararenal SFT, in the absence of clear signs of locoregional infiltration, the recommended extension of resection could be controversial. We hypothesize that, in selected cases, a nephrectomy could be avoided with comparable outcomes [11]. The rarity of pararenal SFT does not allow randomized prospective trials concerning this issue. Preoperative diagnostic efforts must be made to achieve detailed data about its origin in order to plan a tailored surgery. The association of STF and the NF1 gene mutation is intriguing. However, since NF1 gene mutation has not been investigated systematically in STF cases, and the available evidence in the literature data is insufficient, it is hard to hypothesize how a gene mutation might determine the development of a suprarenal neoplasm. More studies are needed to better analyze the relationships between SFT and NF1 gene mutation.

\section{Consent}

Written informed consent was obtained from the patient for the publication of this report and any accompanying images.

\section{Competing interests}

The authors declare that they have no competing interests.

\section{Authors' contributions}

CG: Participated substantially in conception, design, and execution of the study; also participated substantially in the drafting and editing of the manuscript. TE: Participated substantially in conception, design, and execution of the study; also participated substantially in the drafting and editing of the manuscript. GC: Participated substantially in conception, design, and execution of the study. MC: Participated substantially in conception, design, and execution of the study. ED: Participated substantially in conception, design, and execution of the study. MM: Participated substantially in conception, design, and execution of the study. RD: Participated substantially in conception, design, and execution of the study. SG: Participated substantially in conception, design, and execution of the study. GGB: Participated substantially in conception, design, and execution of the study. SL: Participated substantially in conception, design, and execution of the study. All authors read and approved the final manuscript.

\section{Author details}

${ }^{1}$ Department of Anesthesiologic, Surgical and Emergency Science VII Division of General Surgery, Second University of Naples Italy, Via Pansini 5, 80131 Naples, Italy. ${ }^{2}$ Department of Advanced Biomedical Sciences, University of Naples Federico II, Via Pansini 5, 80131 Naples, Italy. ${ }^{3}$ Department of Infectious Diseases, Viral Hepatitis Unit, Second University of Naples, Via Pansini 5, 80131 Naples, Italy.

Received: 24 May 2013 Accepted: 15 March 2014

Published: 7 April 2014

\section{References}

1. Klemperer P, Rabin CB: Primary neoplasm of the pleura: a report of five cases. Arch Pathol 1931, 11:385-412. 
2. Lo Muzio L, Mascolo M, Capodiferro S, Favia G, Maiorano E: Solitary fibrous tumor of the oral cavity: the need for an extensive sampling for a correct diagnosis. J Oral Pathol Med 2007, 36(9):538-542.

3. Morimitsu Y, Nakajima M, Hisaoka M, Hashimoto H: Extrapleural solitary fibrous tumor: clinicopathologic study of 17 cases and molecular analysis of the p53 pathway. APMIS 2000, 108:617-625.

4. Fine SW, McCarthy DM, Chan TY, et al: Malignant solitary fibrous tumor of the kidney: report of a case and comprehensive review of the literature. Arch Pathol Lab Med 2006, 130:857-861.

5. Zinnamosca L, Petramala L, Cotesta D, Marinelli C, Schina M, Cianci R, Giustini S, Sciomer S, Anastasi E, Calvieri S, De Toma G, Letizia C: Neurofibromatosis type 1 (NF1) and pheochromocytoma: prevalence, clinical and cardiovascular aspects. Arch Dermatol Res 2011, 303(5):317-325.

6. Walther MM, Herring J, Enquist E, Keiser HR, Linehan WMJ: von Recklinghausen's disease and pheochromocytomas. Urol 1999, 162(5):1582-1586.

7. Hirato D, Mashiko A, Murata Y, Satoh K, Ichinose T, Takahashi S, Jike T, Sugitani M: Case of solitary fibrous tumor of the kidney: an immunohistochemical and ultrastructural study with a review of the literature. Med Mol Morphol 2009, 42:239-244.

8. Conzo G, Musella M, Corcione F, De Palma M, Ferraro F, Palazzo A, Napolitano S, Milone M, Pasquali D, Sinisi AA, Colantuoni V, Santini L: Laparoscopic adrenalectomy, a safe procedure for pheochromocytoma. A retrospective review of clinical series. Int I Surg 2013, 11(2):152-156.

9. Conzo G, Tricarico A, Belli G, Candela S, Corcione F, Delgenio G, Ferulano GP, Giardiello C, Livrea A, Marzano LA, Porcelli A, Sperlongano P, Vincenti R, Palazzo A, De Martino C, Musella M: Adrenal incidentalomas in the laparoscopic era and the role of correct surgical indications: observations from 255 consecutive adrenalectomies in an Italian series. Can J Surg 2009, 52:281-285.

10. England DM, Hochholzer L, McCarthy MJ: Localized benign and malignant fibrous tumors of the pleura. A clinicopathologic review of 223 cases. Am J Surg Pathol 1989, 13(8):640-658.

11. Conzo G, Sciascia V, Palazzo A, Stanzione F, Della Pietra C, Insabato L, Natella V, Radice L, Santini L: Radiofrequency-assisted partial nephrectomy for metanephric adenoma: a case report and literature review. Surg Innov 2013, 20(1):55-58.

doi:10.1186/1477-7819-12-87

Cite this article as: Conzo et al: Suprarenal solitary fibrous tumor associated with a NF1 gene mutation mimicking a kidney neoplasm: implications for surgical management. World Journal of Surgical Oncology 2014 12:87.

\section{Submit your next manuscript to BioMed Central and take full advantage of:}

- Convenient online submission

- Thorough peer review

- No space constraints or color figure charges

- Immediate publication on acceptance

- Inclusion in PubMed, CAS, Scopus and Google Scholar

- Research which is freely available for redistribution 